GUEST EDITORIAL

\title{
A NATIONAL NEED: REHABILITATION SPECIALIST IN SUBSTANCE ABUSE DISORDER
}

Substance Use Disorder (SUD) is a category of symptoms consisting of behavioral, cognitive, and physiological characteristics arising from the persistent use of the substance despite significant substance-related difficulties in life. Substance usage is growing consistently across the globe as well as in Pakistan. According to one of the studies conducted in 2018; Pakistan has had up to 6.7million drug users, more than 4 million of whom meet the criteria of Substance Use Disorder. Another study also identified a high prevalence of substance use disorders among young adults; making it a national concern.

The high prevalence of drug use in the country is attributed to a rise in social adversities and health disparities. Since Pakistan is a transit route for drugs to other countries, it becomes more vulnerable to substance use. It is difficult to provide a strong monitoring system; therefore there must be a way to minimize the demand and supply. Also previously substance use was perceived to be a moral act and a deficiency in character, now it is changing from a taboo to a social status in some social groups which further increases its consumption among young adults.

SUD is predominantly a chronic, progressive and relapsing condition; prognosis is largely focused on effective multidisciplinary treatment and psycho-social support. Treatment approach to substance use disorders involves multidisciplinary efforts to understand the course of disorder as well as treatment that is often very complicated, time-consuming and long-term. Evidence-based integrated treatment is based on a range of elements such as outreach services, pre-treatment interventions, outdoor treatment, residential treatment, detoxification, recovery and follow-up / after-care programs. Treatment consists of 90 days of residential treatment as per national and international guidelines. A formal recovery program includes a team of recovery practitioners, such as physical therapists, occupational therapists and clinical psychologists. According to one of the study, rate of relapse is $40 \%$ to $60 \%$ which can be reduced with the effective deliverance of rehabilitation services, unfortunately in Pakistan relapse has been observed more than the mentioned statistics, due to lack of awareness about available treatment options and role of rehab professionals.

In Pakistan, the role of rehabilitation professionals is unexplored yet; also there is lack of mental health rehabilitation professionals. According to report generated by WHO and Ministry of Health Pakistan in 2009, only $20 \%$ of substance users receive psychosocial intervention for one or two times. 624 community-based psychiatric inpatient units were available out of which only $1 \%$ is reserved for children and adolescence. The inpatient psychiatric unit has risen in recent years. The number of mental health professionals available is also very less as compared to country population. There is an intense need of cognizance programs by the government and educational institutes to train more mental health care professionals and establish guidelines for community support and engagement.

The contribution of rehab professionals is beyond mental and physical health improvement. Effective treatment from multidisciplinary rehabilitation team will result relapse control, regaining of physical strength, healthy routine and integrating back to functional life making the individual productive and contributing member of society.

Each member of Multidisciplinary rehabilitation team plays unique and vital role in rehabilitation of substance users. Clinical psychologist help individuals with substance use disorder to manage thoughts, emotions and behaviors, also restructure relationship with family and peers. Occupational therapy improves the quality of life of recovering individuals from SUD and work on relapse prevention. It is accomplished by empowering individuals to recognize their meaningful occupations and occupational roles while supporting them regain their previous occupational patterns. Sports rehabilitation, physical fitness and exercises by physical therapist promote the body fitness and collaborate with detoxification of body. Mindfulness along with life style management program plays significant role in SUD rehabilitation.

Vocational training and job placement is another main aspect of rehabilitation of SUDs. Under the objective of vocational challenges and solutions, professionals such as Occupational Therapist and Social workers provide ways in which the person returns to their previous life roles and find new vocational opportunities. It is achieved by helping them learn money management, completing daily household chores as well as finding and keeping a job by assessing occupational engagement and barriers. 
Community awareness and Family education has vital role. Supportive social circle accelerates the treatment process. Family centered approach by rehab professionals has massive impact on the caregiver burnout and advocacy which brings better strategies in coping with individual with substance use disorder at home.

Rehabilitation centers for SUD in Pakistan lack skill full professional. Many evidence base practices have been established in this field and proved that rehab specialist arises as an indispensable member of holistic team at drug rehabilitation. The rehab professional develops significant proximity with the individual suffering from SUD since the zing among the professionals assist the patient in regaining the health and wellness. This gap can be met by promoting community awareness programs and more scientific researches.

\section{Dr. Nooreen Begum}

Senior Clinical Psychologist/ Rehabilitation Incharge

Model Addiction Treatment and Rehabilitation Center,

Anti Narcotics Force, Karachi.

DOI: 10.36283/pjr.zu.9.2/001 